\title{
Making Better Decisions: From Measuring to Constructing Preferences
}

\author{
Eric J. Johnson, Mary Steffel, and Daniel G. Goldstein \\ Columbia University
}

\begin{abstract}
The authors examine how a constructive preferences perspective might change the prevailing view of medical decision making by suggesting that the methods used to measure preferences for medical treatments can change the preferences that are reported. The authors focus on 2 possible techniques that they believe would result in better outcomes. The 1st is the wise selection of default options. Defaults may be best applied when strong clinical evidence suggests a treatment option to be correct for most people but preserving patient choice is appropriate. The 2 nd is the use of environments that explicitly facilitate the optimal construction of preferences. This seems most appropriate when choice depends on a patient's ability to understand and represent probabilities and outcomes. For each technique, the authors describe the background and literature, provide a case study, and discuss applications.
\end{abstract}

Keywords: constructive preferences, decision analysis, defaults, decision environments, affective forecasting

Many patients newly diagnosed with Hodgkin's Disease (a cancer of the lymphatic system) must choose between the following chemotherapy treatments:

1. The clinical standard, ABVD, which presents a longer but less intense experience. Treatment typically lasts for 6 months, with chemotherapy sessions occurring once every 2 weeks.

2. The more recent therapy, Stanford V, which presents a briefer but more intense experience, with potential longterm implications. Treatment typically lasts for 3 months, with chemotherapy sessions occurring weekly. These two regimens offer different experience in the short term and different risks in the long term.

Decision theory suggests that this choice involves accessing how one feels about the duration and intensity of the treatments and making the appropriate tradeoffs between the costs and benefits of a longer, less intense treatment and those of a shorter, more intense treatment - a process facilitated, perhaps, by decision analysis, a technique that explicitly represents and examines relevant information for a decision and the likelihood of obtaining particular outcomes.

Now consider the following, less serious questions: How much would you pay to rid yourself of one migraine headache? What would you pay for a $108 / 130$ chance at winning $\$ 33$ ? How about a 1998 Cotes du Rhone with a Wine Advocate Rating of 86? Or a 1996 Hermitage Jaboulet "La Chapelle" with a 96 rating?

Eric J. Johnson, Mary Steffel, and Daniel G. Goldstein, Center for Decision Sciences, Columbia University.

This research was supported by Grant SES-0352062 from the National Science Foundation. We thank Ellen Peters and Kevin McCaul for their many helpful comments.

Correspondence concerning this article should be addressed to Eric J. Johnson, Columbia Business School, Columbia University, 514 Uris Hall, New York, NY 10027. E-mail: ejj3@ columbia.edu
Like the treatment question, these questions require one to make tradeoffs, but research suggests that even these simpler questions are not easy to answer. The first, used by Chapman and Johnson (1999), is influenced by asking people to compare the amount they would pay with a randomly chosen number or anchor. The second is influenced by whether the probability is expressed as a hard-tocomprehend fraction, as noted above, or as its equivalent decimal probability .8 (Johnson, Payne, \& Bettman, 1988). Finally, the wine example has been used to show that arbitrary anchors (based on respondents' social security numbers) influence prices, even when the goods are priced by an auction (Ariely, Loewenstein, \& Prelec, 2003) by people (Stanford MBA students) who have a modicum of economic sophistication.

Why are even these "easy" questions so hard? Perhaps because people do not have well defined preferences for these particular questions, as decision analysis would assume. Rather, people may construct preferences at the time the question is asked. For many common decisions, this seems to be the case. The major evidence is that significant changes in choices occur in the presence of what should be meaningless changes in the task. In the examples above, the presence of a randomly chosen anchor or the representation of a number as a fraction or a decimal should not affect an existing preference, but empirically, they do. The effects can be quite substantial. In the headache example above, respondents were willing to pay $\$ 10$ for the medicine when there was no anchor, but they were willing to pay an average of $\$ 3.20$ when presented with a low anchor of $\$ 1.90$ and $\$ 17.80$ when presented with a high anchor of $\$ 19$

Now consider difficult, infrequent medical decisions. Recent developments have brought decision theory to the bedside by using decision analysis models that are based on expected utility theory. These are intended to support the decision process by providing a formal and explicit framework for assessing preferences and making choices. Among the most common preference assessment methods used in decision analysis are the time tradeoff (TTO), rating scale (RS) and standard gamble (SG). TTO asks patients how many years of living in a treatment-related health state they would trade for a shorter life expectancy in perfect health, RS asks 
patients to rate health states on a scale ranging from death to perfect health, and SG asks patients how much risk they would be willing to accept to be restored to perfect health. As they are measuring the same underlying construct of preferences, we expected these three methods to produce similar results.

Whereas formal models of preference assessment are based on the assumption that individuals have clear, stable, well ordered preferences, decision-analytic treatment recommendations often vary with the method of elicitation, much as did the prices for headache remedies, gambles, and wine. Elkin, Cowen, Cahill, Steffel, and Kattan (2004) compared TTO and RS values, elicited from a sample of 63 men aged 55-75 years, in a decision analytic model for watchful waiting versus surgical therapy for prostate cancer. RS and TTO values differed in their treatment recommendation for $21 \%-52 \%$ of participants, depending on the severity of prostate cancer assumed. Only 2 of the 63 participants gave TTO and RS values that exhibited identical rank ordering of health states. Read, Quinn, Berwick, Fineberg, and Weinstein (1984) compared RS and SG values, elicited from a sample of 60 physicians, in a decision model of surgical versus medical therapy for coronary heart disease. For $60 \%$ of the participants, the treatment recommended by the decision model varied with the method of preference assessment.

Such decisions, it seems, are not about uncovering existing values - they are about inventing them or, in terms that are common in behavioral decision theory, constructing them. The notion of constructive preferences shifts our approach from trying to measure already-established preferences to facilitating their construction. It suggests that the methods used to measure preferences can change the preferences that are reported. Most important, the idea of constructive preferences changes the way in which researchers may want to aid medical decisions.

The idea of constructive preferences suggests two different interventions. The first is to structure the decision environment in a way that will produce decisions that are most likely to have better outcomes. An example of this is the wise selection of default options. The second is to try to structure the decision environment in a way that will improve the ability of the patient to understand and represent probabilistic outcomes. Examples of this approach include tools that allow people to explicitly represent probabilistic outcomes and sample among them.

In the remainder of the present article, we examine how a constructive preferences perspective might change researchers' view of medical decision making, focusing on these two possible approaches. For each approach, we describe the background literature, provide a case study, and discuss applications.

\section{Defaults and Decisions}

\section{Defaults Matter}

A major and, perhaps to some, surprising conclusion is that identifying one option as a default (i.e., the option selected if no active decision is made) has a sizable impact on what is chosen, even for economically or socially significant decisions.

One set of demonstrations involves inadvertent "natural experiments," in which governments, companies, and public agencies randomly assign people to one program and give them a choice between that option and another alternative. For example, default effects occur in the choice of health care plans (Samuelson \&
Zeckhauser, 1988) and the choice between privacy policies for personal information on the Internet (Bellman, Johnson, \& Lohse, 2001; Johnson, Bellman, \& Lohse, 2002). In most cases, the majority of people choose the default option to which they are assigned, even when this assignment is random.

This occurs even when the stakes are high. In one example, New Jersey and Pennsylvania gave buyers of auto insurance a choice between a more expensive plan that provided the right to sue for pain and suffering and a significantly less expensive plan that covered the medical costs of the insured but removed the right to sue for difficult-to-prove "pain and suffering" claims. New Jersey drivers were given the limited right to sue by default, whereas Pennsylvania drivers had the opposite default, that is, the full right to sue. Of note is that $79 \%$ of New Jersey drivers preferred the limited right to sue, whereas $70 \%$ of Pennsylvania drivers preferred the opposite plan. A psychological study, in which people were assigned one of the two plans by default, confirmed this: The full right to sue was chosen $53 \%$ of the time when it was the default but only $23 \%$ of the time when it was not (Johnson, Hershey, Meszaros, \& Kunreuther, 1993).

Why do defaults make such a difference? Different studies have identified three potential causes of default effects. First, people may interpret defaults as suggestions or recommendations (Johnson et al., 1993). Second, people may wish to avoid the effort and cost of changing from a default, preferring to accept a default to making an active, effortful choice. Many people show an omission bias, that is, a tendency to judge acts that lead to harmful outcomes as worse than omissions that are equally or more harmful (Baron \& Ritov, 1994). Finally, people may feel reluctant to give up the status quo, represented by the default option. They experience loss aversion; that is, they experience losses more intensely than equivalent gains (Tversky \& Kahneman, 1991). Therefore, changes in the default may result in different tradeoffs and different choices.

\section{Case Study: Organ Donation}

The role of default effects, and their importance in a health setting, is illustrated in a study of organ donation (see Johnson \& Goldstein, 2003, for details). We first examined the role of defaults by using an online experiment. We asked 161 respondents whether they would be donors using one of three default conditions: an opt-in condition in which the default was not to be an organ donor, an opt-out condition in which the default was to be a donor, and a neutral condition with no prior default. The default had a dramatic impact, with revealed donation rates being about twice as high when opting out as when opting in. As is shown in Figure 1, the opt-out condition did not differ significantly from the neutral condition. Only the opt-in condition, the current practice in the United States, was significantly lower.

As there are many factors that might produce different effects in the real world, we examined the rate of agreement to become a donor across European countries with different defaults. As is shown in Figure 2, defaults make a large difference, with the four opt-in countries on the left having lower rates than the six opt-out countries on the right. The result is surprisingly strong: The two distributions have no overlap, and nearly 60 percentage points separate the two groups. We think these effects are larger than those in our questionnaire because the cost of changing from the default is higher, involving filling out forms, making phone calls, or sending mail. 


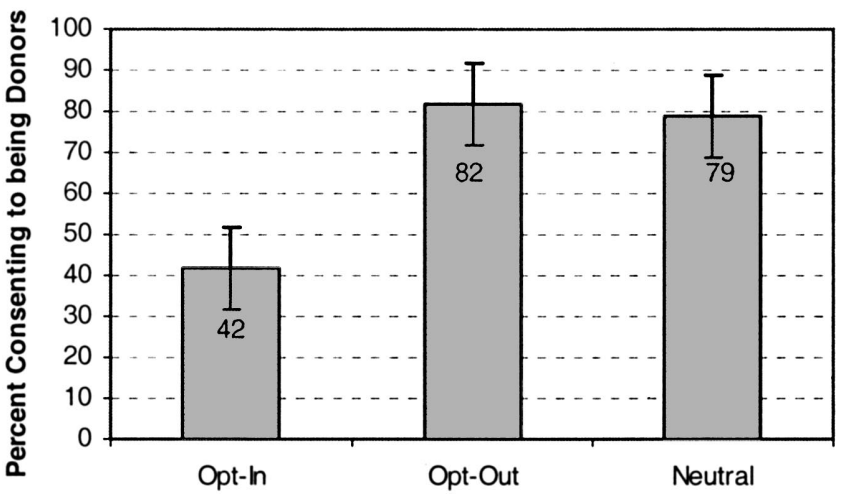

Figure 1. Effective consent rates, online experiment, by default. From "Medicine: Do Defaults Save Lives?" by E. J. Johnson and D. Goldstein, November 21, 2003, Science, 302, p. 1338. Copyright 2003 by the American Association for the Advancement of Science. Reprinted with permission.

We examined the actual number of caderveric donations made per million on a slightly larger list of countries between 1991 and 2001, controlling for variables known to affect donation rates (see Gimbel, Strosberg, Lehrman, Gefenas, \& Taft, 2003). Whereas there are no differences across years, there is a strong effect of the default: When donation is the default, there is a significant increase in donation, increasing from 14.1 to 16.4 , a $16.3 \%$ increase. Using similar techniques, but looking only at 1999 for a broader set of countries, another similar study reports an increase from 10.8 to 16.9, a 56.5\% increase (Gimbel et al., 2003).

\section{Applications}

What does this mean for medical decision making? This research suggests several implications: First, a cancer patient might interpret a default treatment regimen as a recommendation and simply assume that the doctor knows best rather than seek out alternative treatment options. Second, a patient might avoid mak- ing an active decision about a treatment regimen because considering the possible outcomes is scary and stressful or because researching all possible options is taxing and complex. Or, due to omission bias, a patient might prefer to stick with a standard treatment option and risk omitting a better option rather than to actively choose a different option and risk committing a mistake. Third, due to loss aversion, a patient might feel reluctant to give up the default, or status quo, for another option.

To return to our previous example, different defaults present the patient with two different frames. The Hodgkin's disease patient who sees ABVD as the default may view changing to Stanford V as a tradeoff between a gain (less time in treatment) and a loss (more discomfort during treatment). For a patient who sees Stanford $\mathrm{V}$ as the default, changing treatments changes the tradeoff: gaining comfort during treatment but losing months of healthy living.

Most important, perhaps, is the realization that defaults will affect choices. If there is strong clinical evidence suggesting an appropriate therapy, then perhaps that therapy should be presented as the default. The patient still does make a choice, but the choice of default serves to represent the best medical judgment without overriding the patient's preferences. Arguments that this represents a paternalistic treatment of patients must encounter two realities: The first is that all choices have a no-action default. The second is that any option is more likely to be adopted when it is the default. In those circumstances where current knowledge suggests that one option is more likely than another to produce a better outcome, then having an appropriate default seems wise.

\section{Environments for Constructing Preferences}

\section{Environments Matter}

Whereas defaults are a useful tool when there is a clear idea of what might be a better option, there are certainly applications where this is not the case. Our goal in these cases may be to structure the decision environment in such a way as to help decision makers do a better job of understanding the possible

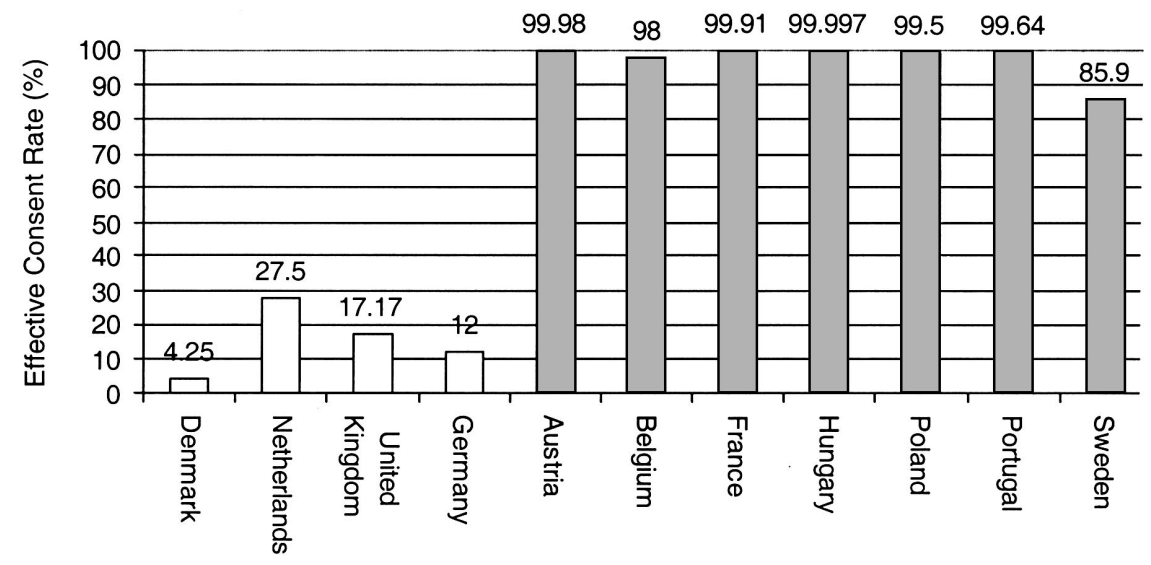

Figure 2. Effective consent rates, by country. The four leftmost bars are explicit consent (opt-in), and the seven rightmost bars are presumed consent (opt-out). From “Medicine: Do Defaults Save Lives?" by E. J. Johnson and D. Goldstein, November 21, 2003, Science, 302, p. 1338. Copyright 2003 by the American Association for the Advancement of Science. Reprinted with permission. 
outcomes of a decision and representing the likelihood of obtaining those outcomes. Unlike research in default effects, efforts here are much less well developed. Although we know that many different aspects of the decision environment change stated choices, we know much less about which aspects lead to choices that are, in some sense, more satisfying. Nonetheless, results from research on judgment and decision making from the last 25 years have identified principles of information representation that seem to facilitate reasoning about risk in accordance with a variety of normative standards. We speculate that principles such as the following may help in constructing environments for medical decision making, and we hope to see future research look in this direction.

Frequency versus numerical representations of probability. Three ways in which probabilities can be displayed-fractions, decimals, and relative frequencies - though mathematically equivalent, are often psychologically quite different in the computations they facilitate (Feynman, 1967). Probabilities expressed not in a stated numerical format (such as .002) but in a frequency format (such as 2 in 1,000) have been shown to improve probabilistic reasoning on a number of problem-solving tasks. For an excellent review, see Gigerenzer (1994; see also Hoffrage \& Gigerenzer, 1998; Hoffrage, Kurzenhäuser, \& Gigerenzer, 2005; Kahneman \& Lovallo, 1993; Sedlmeier, 1997). If frequency representations were better understood, we would predict them to be helpful in doctor-patient interactions when communicating the choice between treatment options and in the interpretation of test results.

Experienced versus stated representations of probability. Stated probabilities are psychologically different from those that are experienced first hand or in simulation. Visually experiencing chance outcomes enables an automatic and unconscious kind of probability learning (Christensen-Szalanski \& Beach, 1982; Gigerenzer \& Hoffrage, 1995; Hasher \& Zacks, 1984) and helps people achieve an intuitive grasp of the choices before them (Evans, 1989, p. 118). If the goal is to improve the construction of preferences, a fruitful direction may be to use visual simulations to communicate information about the magnitude and cumulative effects of risk, but more research in this direction is needed to fully understand how experienced probabilities are interpreted (see Hertwig, Barron, Weber, \& Erev, 2004; Weber, Shafir, \& Blais, 2004).

Integrated versus isolated representations. Another significant finding of decision research is that decisions made in isolation can result in inconsistent choices. Examples of this include demonstrations that two sequential decisions (e.g., gambles) can result in different choices than a single integrated choice (Thaler \& Johnson, 1990) and that choices made about isolated risks can lead to undesirable outcomes when those risks are aggregated (Kahneman \& Lovallo, 1993). We endorse representations that explicitly portray how sets of decisions are interrelated. In such frameworks, one decision cannot be explored without seeing its effect on other outcomes. In the medical domain, this might suggest expressing the relationship between repeated risks and their cumulative impact on life expectancy. For instance, a patient may choose not to cease a dangerous behavior that has a $1 \%$ chance of fatality in a given year. However, after learning that this risk aggregates to a greater than $25 \%$ chance of death in 30 years, he or she may feel differently.

\section{Case Study: Retirement Savings}

To show how the three principles noted above have been built into a preference-construction aid, we provide an example from another important domain: constructing risk-return preferences for retirement investments. We have created an environment called Distribution Builder for constructing preferences, the interface of which is shown in Figure 3. The Distribution Builder allows people to construct probability distributions of the monetary outcomes they would like to achieve in retirement, constrained by the size of their investment and the returns of real markets.

In Figure 3, monetary outcomes are expressed by the rows of the vertical axis, and probabilities are expressed by the number of markers placed in each row. The 41 rows represent income in retirement, expressed as a percentage of preretirement income, and range from $0 \%$ to $200 \%$. The lowest row has three markers stacked at $65 \%$, visually representing a .03 probability of earning $65 \%$ of preretirement income while in retirement. By clicking and dragging a marker, the user can move that one probability marker and all those to its right. By moving the mouse, users can quickly and easily alter the probabilities assigned to various outcomes. On the upper left-hand side of Figure 3 is a bar, termed the cost meter. Underlying the Distribution Builder is a sophisticated piece of financial engineering that calculates, in real time, the cost of proposed distributions. As investors develop potential distributions, they are shown the cost of the distribution and are given a target cost that they should not exceed.

How does this interface implement the principles we described earlier? First, probabilities are not stated but, rather, expressed through the visual frequency representation of the 100 markers. Second, while constructing and submitting distributions in a training session, users visually experience random drawings from them, enabling them to engage in probability learning through simulation. Finally, because no one marker can be moved without affecting the cost of the entire distribution, no part of the overall decision can be made in isolation. The cost meter serves the goal of presenting an integrated representation quite well. If they exceed the budget, they must then decide how to remedy the problem and are not allowed to submit any distribution that exceeds their cost budget.

As an example of the kinds of preferences that can be constructed with this tool, we present, in Figure 4, an aggregate preferred distribution of income in retirement by respondents to an online study (Goldstein, Johnson, \& Sharpe, 2004). As far as we know, this is the first investigation that has asked people to construct preferences for cost-constrained probability distributions. Exploring distributions interactively was simply not practical before the now-ubiquitous graphical user interfaces of computers. We speculate that similar technologies can be developed for medical decision making, allowing patients to explore treatment options and the associated cost-benefit tradeoffs.

\section{Applications}

Whereas research in constructing environments is just beginning, we hope that the Distribution Builder might be adapted for describing therapy choices. One example might be in aiding decisions among breast cancer treatments, particularly in women who have undergone genetic testing for presence of the BRCA1/ BRCA2 mutation carriers (Schwartz, Peshkin, Tercyak, \& 


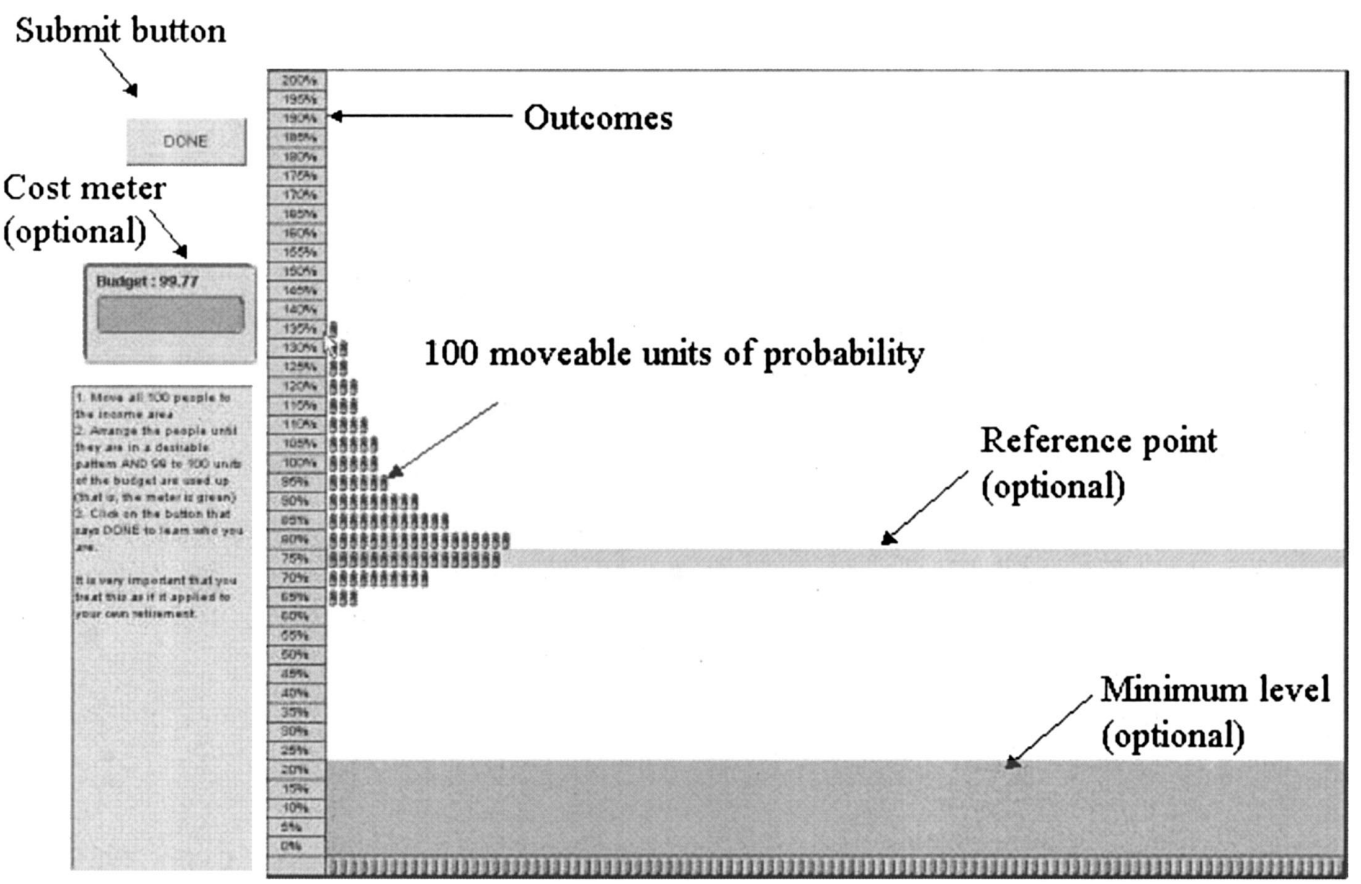

Figure 3. The Distribution Builder interface. Using moveable units of probability, users can create arbitrarily shaped discrete probability distributions over numerous outcomes (on the vertical axis).

Valdimarsdottir, 2004). Side effects could be expressed on the rows of the interface, and the number of people stacked at each row could reflect the probability of experiencing an outcome. Patients could construct their own preference profile and determine which of the various treatment options comes closest to their preferences. Patients could simulate a number of random drawings to get an idea of how many times a particular side effect may result in a 10 -year period. In another application, the interface rows could be used to express years of life expectancy given various treatment options, and the number of people stacked at each row could express the probability of that life expectancy being realized.
Though contemplating the probability distribution of one's life expectancy is grim, some patients may appreciate being able to understand the risks for the sake of planning for the future of their families.

\section{Conclusion}

In this article, we have explored how a constructive preferences perspective to medical decision making shifts the focus from measuring preferences to facilitating preference construction. We suggest two techniques that we think are helpful in the face of

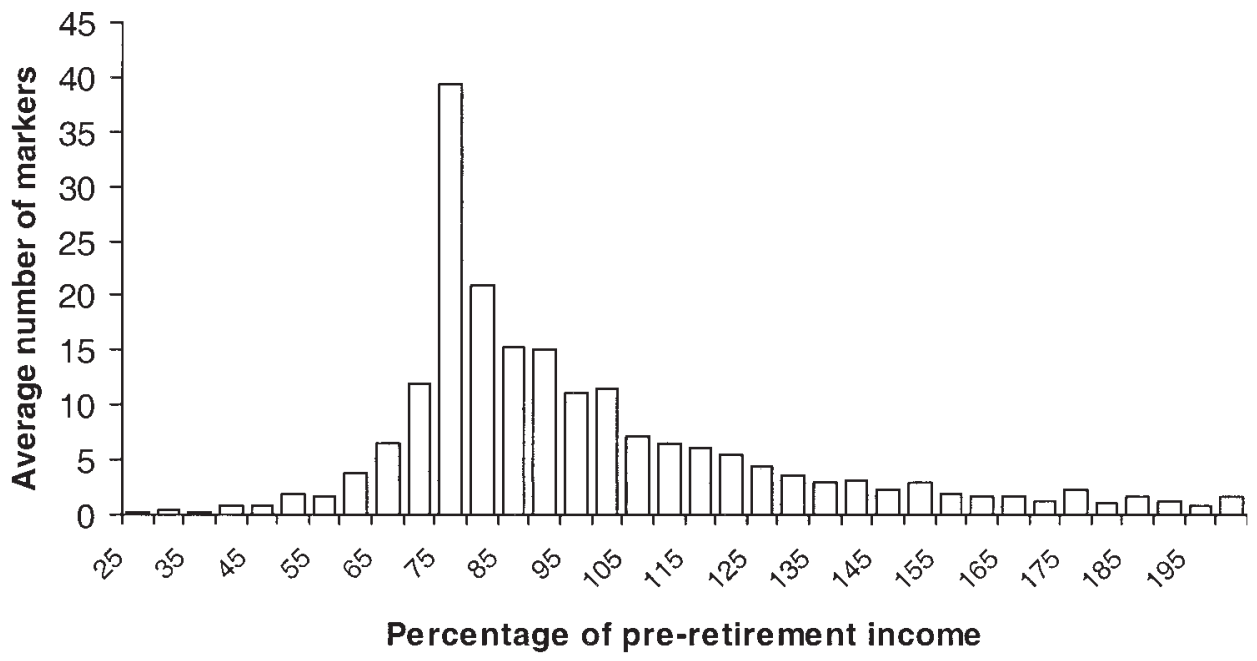

Figure 4. The distribution of the average investor, created by averaging the number of markers at each wealth level across all participants. 
preference construction: The first is the wise selection of default options. Defaults may be best applied when strong clinical evidence suggests a treatment option to be correct for most people but preserving patient choice is appropriate. The second is the use of environments that explicitly facilitate the construction of preferences. This seems most appropriate when choice depends on a patient's ability to understand and represent probabilistic outcomes.

The two approaches that we prescribe differ in applicability to cancer decision making, obviously, as the first requires that we have some idea of what outcomes are more likely to produce good results. This raises an obvious problem: Should the designer of the decision environment affect preferences? The idea itself suggests a paternalistic relationship with the patient (for further review, see Camerer, Issacharoff, Loewenstein, O’Donoghue, \& Rabin, 2003; Thaler \& Sunstein, 2003). We argue that there is often no alternative. In the case of defaults, all choices have a no-action default, and any option is more likely to be adopted when it is the default. Likewise, no choice takes place in a vacuum, and ignoring the fact that the environment affects the choice represents a missed opportunity.

In today's society, decision counseling entails more than just presenting patients with information about their options and assessing their preferences. It also encompasses how the information is presented and how the decision-making process is facilitated. We admit that it is certainly possible to imagine decisions in which people do have existing, well defined preferences that are robust to the way information is framed and preferences are elicited. However, we emphasize that, for many patients facing difficult, unfamiliar medical decisions, presentation makes a great deal of difference, and the behavioral consequences of otherwise-identical information may often depend on how it is presented. For cancer patients, the manner in which preferences are constructed may influence critical choices such as what preventative measures to take, whether to seek diagnostic information, and which treatment to choose.

\section{References}

Ariely, D., Loewenstein, G., \& Prelec, D. (2003). "Coherent arbitrariness": Stable demand curves without stable preferences. Quarterly Journal of Economics, 118(1), 73-105.

Baron, J., \& Ritov, I. (1994). Reference points and omission bias. Organizational Behavior and Human Decision Processes, 59, 475-498.

Bellman, S., Johnson, E. J., \& Lohse, G. L. (2001). To opt-in or opt-out? It depends on the question. Communications of the ACM, 44(2), 25-27.

Camerer, C., Issacharoff, S., Loewenstein, G., O’Donoghue, T., \& Rabin, M. (2003). Regulation for conservatives: Behavioral economics and the case for "asymetric paternalism." University of Pennsylvania Law Review, 151, 1211-1254.

Chapman, G. B., \& Johnson, E. J. (1999). Anchoring, activation, and the construction of values. Organizational Behavior and Human Decision Processes, 79(2), 115-153.

Christensen-Szalanski, J. J. J., \& Beach, L. R. (1982). Experience and the base-rate fallacy. Organizational Behavior and Human Performance, 29, 270-278.

Elkin, E. B., Cowen, M. E., Cahill, D., Steffel, M., \& Kattan, M. W. (2004). Preference assessment method affects decision analytic recommendations: A prostate cancer treatment example. Medical Decision Making, 24(5), 504-510.
Evans, J. B. T. (1989). Bias in human reasoning: Causes and consequences. Hillsdale, NJ: Erlbaum.

Feynman, R. (1967). The character of physical law. Cambridge, MA: MIT Press.

Gigerenzer, G. (1994). Why the distinction between single-event probabilities and frequencies is relevant for psychology (and vice versa). In G. W. P. Ayton (Ed.), Subjective probability (pp. 129-161). New York: Wiley.

Gigerenzer, G., \& Hoffrage, U. (1995). How to improve Bayesian reasoning without instruction: Frequency formats. Psychological Review, 102(4), 684-704.

Gimbel, R. W., Strosberg, M. A., Lehrman, S. E., Gefenas, E., \& Taft, F. (2003). Presumed consent and other predictors of cadaveric organ donation in Europe. Progress in Transplantation, 13(1), 17-23.

Goldstein, D. G., Johnson, E. J., \& Sharpe, W. F. (2004). Measuring consumer risk-return tradeoffs. Manuscript submitted for publication.

Hasher, L., \& Zacks, R. T. (1984). The automatic processing of fundamental information: The case of frequency of occurrence. American Psychologist, 39, 1372-1388.

Hertwig, R., Barron, G., Weber, E. U., \& Erev, I. (2004). Decisions from experience and the effect of rare events in risky choice. Psychological Science, 15, 534-539.

Hoffrage, U., \& Gigerenzer, G. (1998). Using natural frequencies to improve diagnostic inferences. Academic Medicine, 73, 538-540.

Hoffrage, U., Kurzenhäuser, S., \& Gigerenzer, G. (2005). Understanding the results of medical tests: Why the representation of statistical information matters. In R. Bibace, J. D. Laird, K. L. Noeller, \& J. Valsiner (Eds.), Science and medicine in dialogue: Thinking through particulars and universals (pp. 83-98). Westport, CT: Praeger.

Johnson, E. J., Bellman, S., \& Lohse, G. L. (2002). Defaults, framing, and privacy: Way opting in-opting out. Marketing Letters, 13(1), 5-15.

Johnson, E. J., \& Goldstein, D. (2003, November 21). Medicine: Do defaults save lives? Science, 302, 1338-1339.

Johnson, E. J., Hershey, J., Meszaros, J., \& Kunreuther, H. (1993). Framing, probability distortions, and insurance decisions. Journal of Risk and Uncertainty, 7, 35-51.

Johnson, E. J., Payne, J. W., \& Bettman, J. R. (1988). Information displays and preference reversals. Organizational Behavior \& Human Decision Processes, 42(1), 1-21.

Kahneman, D., \& Lovallo, D. (1993). Timid choices and bold forecasts: A cognitive perspective on risk taking. Management Science, 39, 17-31.

Read, J. L., Quinn, R. J., Berwick, D. M., Fineberg, H. V., \& Weinstein, M. C. (1984). Preferences for health outcomes: Comparison of assessment methods. Medical Decision Making, 3, 315-329.

Samuelson, W., \& Zeckhauser, R. (1988). Status quo bias in decisionmaking. Journal of Risk and Uncertainty, 1, 7-59.

Schwartz, M. D., Peshkin, B. N., Tercyak, K., \& Valdimarsdottir, H. (2004). Decision making and decision support for hereditary breastovarian cancer susceptibility. Health Psychology, 24(Suppl. 4), S78S84.

Sedlmeier, P. (1997). BasicBayes: A tutor system for simple Bayesian inference. Behavior Research Methods, Instruments, \& Computers, 29, $328-336$.

Thaler, R., \& Johnson, E. J. (1990). Gambling with the house money and trying to break even: The effects of prior outcomes on risky choice. Management Science, 36(6), 643-660.

Thaler, R. H., \& Sunstein, C. (2003). Libertarian paternalism is not an oxymoron. University of Chicago Law Review, 70, 1159-1202.

Tversky, A., \& Kahneman, D. (1991). Loss aversion in riskless choice: A reference-dependent model. Quarterly Journal of Economics, 106(4), $1039-1061$.

Weber, E. U., Shafir, S., \& Blais, A.-R. (2004). Predicting risk sensitivity in humans and lower animals: Risk as variance or coefficient of variation. Psychological Review, 111, 430-445. 